\title{
Role of the intermediate state in the photoinduced process of Co-Fe cyanide
}

\author{
Y. Moritomo, F. Nakada, and H. Kamioka \\ Department of Physics, University of Tsukuba, Tsukuba 305-8571, Japan \\ T. Hozumi and S. Ohkoshi \\ Department of Chemistry, University of Tokyo, Tokyo 113-0033, Japan
}

(Received 15 March 2007; revised manuscript received 20 April 2007; published 18 June 2007)

\begin{abstract}
The dynamics of the photoinduced phase transition has been investigated for the Co-Fe cyanide film, $\mathrm{Na}_{0.35} \mathrm{Co}\left[\mathrm{Fe}(\mathrm{CN})_{6}\right]_{0.79} \cdot 3.7 \mathrm{H}_{2} \mathrm{O}$, by means of the transient absorption spectroscopy. We observed two characteristic transient bands: short-lived intermediate band (I band) at $\sim 1.9 \mathrm{eV}$ and long-lived photoinduced band (PI band) at $\sim 2.5 \mathrm{eV}$. We have found that the intensity of the I band transfers to the PI band as time exceeds, and interpreted the behavior in terms of the intersystem crossing from the photocreated low-spin $\mathrm{Co}^{2+}$ species to the high-spin species. We further have found that the excitation spectra of these bands are qualitatively different.
\end{abstract}

DOI: 10.1103/PhysRevB.75.214110

PACS number(s): 68.18.Jk, 42.50.Md

The photoinduced phenomena ${ }^{1}$ have been extensively investigated both from the fundamental and technical points of view. In an extreme case, the photoexcitation causes a macroscopic structural change, or the so-called photoinduced phase transition (PIPT). So far, a long list of researchers reported the permanent PIPTs in the spin-crossover complexes, ${ }^{2-5}$ the cyano-bridged metal complexes, ${ }^{6-11}$ the mixed-valence gold complex $\mathrm{Cs}_{2} \mathrm{Au}_{2} \mathrm{Br}_{6},{ }^{12}$ the iodinebridged binuclear Pt compounds, ${ }^{13}$ the organic radical crystal TTTA (1,3,5-trithia-2,4,6-triazapentalenyl), ${ }^{14}$ the polydiacetylene crystal, ${ }^{15}$ and so on. These materials accompany significant changes of the physical properties on the PIPT, and hence, may be utilized as opto-functional devices. In addition, there should be an important physics in the process of the PIPT. Especially, we should answer how the single photoexcitation expands into a macroscopic phase. The irreversibility of the PIPT, however, prevents the detailed investigation on the dynamics of the PIPT. Recently, Yamauchi et al. ${ }^{16}$ overcame the difficulty and reported a dynamics of the PIPT in the Co-Fe cyanide: their idea is that the relaxation time $\left(\tau_{\mathrm{PI}}\right)$ of the photoinduced metastable state becomes faster at high temperature, which enable us to perform a time-resolved experiment.

The Co-Fe cyanides, whose chemical formula is $A_{x} \mathrm{Co}\left[\mathrm{Fe}(\mathrm{CN})_{6}\right]_{y} \cdot z \mathrm{H}_{2} \mathrm{O}(A=\mathrm{Na}, \mathrm{K}, \mathrm{Rb}, \mathrm{Cs})$, have been attracting renewed interest of the material scientists, because they show the photoinduced magnetization ${ }^{6,7}$ as well as the photoinduced structural change. ${ }^{10}$ For example, Sato et al. ${ }^{6}$ reported enhancement of the magnetization in $\mathrm{K}_{0.14} \mathrm{Co}\left[\mathrm{Fe}(\mathrm{CN})_{6}\right]_{0.71} \cdot 4.93 \mathrm{H}_{2} \mathrm{O}$ by irradiation of a red light $(660 \mathrm{~nm})$ at $5 \mathrm{~K}$ and suppression of the magnetization by irradiation of a blue light $(450 \mathrm{~nm})$ at $5 \mathrm{~K}$. Crystallographically, the Co-Fe cyanides belong to the face-centered cubic $(F m \overline{3} m ; Z=4)$, in which $\mathrm{Co}$ and $\mathrm{Fe}$ ions form a rock-salttype (NaCl-type) network bridged by the cyano $\left(\mathrm{CN}^{-}\right)$moieties. This compound has a large amount of the $\mathrm{Fe}(\mathrm{CN})_{6}$ vacancy, which significantly influences the magnetic and electronic properties. ${ }^{11}$ This is probably because the energy levels of the $d$-electron configuration is modified by the surrounding vacancies.
In Fig. 1, we show the electronic configurations of the neighboring Co-Fe pair. The electronic configuration at the ground state (LS phase) is $\mathrm{Co}^{3+}\left(t_{2 \mathrm{~g}}^{6}\right)-\mathrm{Fe}^{2+}\left(t_{2 \mathrm{~g}}^{6}\right)$. The LS phase can be thermally and/or phototransferred to the PI phase with $\mathrm{Co}^{2+}\left(t_{2 \mathrm{~g}}^{5} e_{\mathrm{g}}^{2}\right)-\mathrm{Fe}^{3+}\left(t_{2 \mathrm{~g}}^{5}\right)$ configuration. Here, we stress that the dipole-allowed charge transfer creates the LS $\mathrm{Co}^{2+}$ species (I state in Fig. 1), not the high-spin (HS) $\mathrm{Co}^{2+}$ species. Then, the intersystem crossing process in the $\mathrm{Co}^{2+}$ site would be indispensable to complete the PIPT process. By means of the transient spectroscopy, Yamauchi et al. ${ }^{16}$ observed two characteristic absorption bands, and ascribed them to the PI phase and the I state. The interrelation between the two bands, however, remains unclear.

In this paper, our attention was focused on the interrelation between the I state and the PI phase. Especially, we observed a flow-in (flow-out) behavior in the temporal evolution of the site density $n_{\mathrm{PI}}$ of the PI phase, which is quantitatively reproduced by a rate equation including three energy levels, i.e., the LS phase, the $I$ state, and the PI phase. This clearly indicates that the I state, which was directly

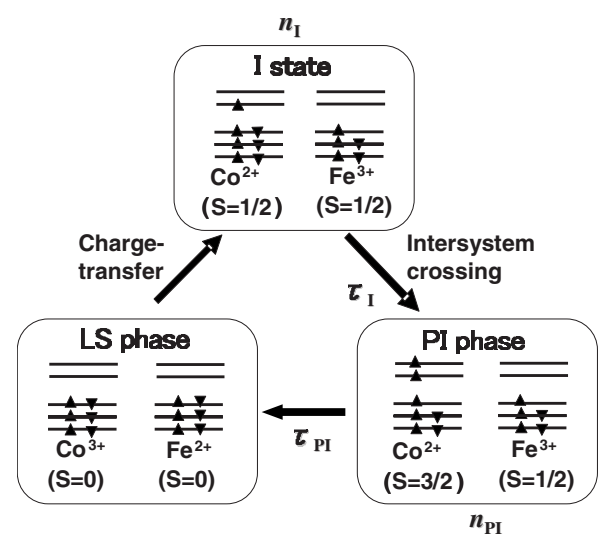

FIG. 1. Electronic configurations of the neighboring Co-Fe pair in the low-spin phase (LS phase), photoinduced metastable phase (PI phase), and the intermediate state (I state). $\tau_{\mathrm{I}}$ and $\tau_{\mathrm{PI}}$ are the relaxation times of the I state and PI phase, respectively. $n_{\mathrm{I}}$ and $n_{\mathrm{PI}}$ are the site densities of the I state and the PI phase, respectively. 


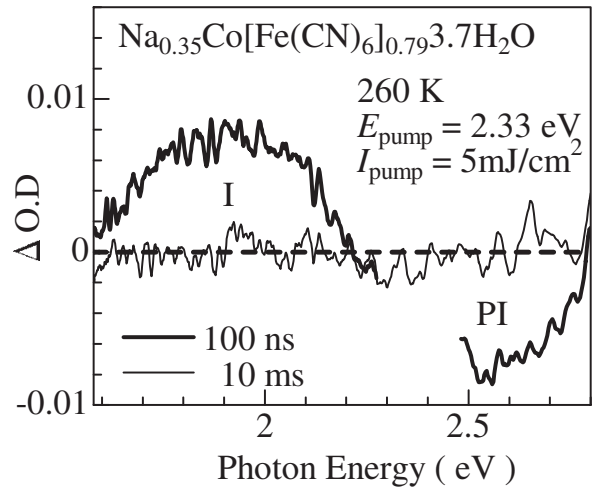

FIG. 2. Differential absorption spectra of $\mathrm{Na}_{0.35} \mathrm{Co}\left[\mathrm{Fe}(\mathrm{CN})_{6}\right]_{0.79} \cdot 3.7 \mathrm{H}_{2} \mathrm{O}$ at $260 \mathrm{~K}$. The pulse energy and the photon energy of the excitation light were $5 \mathrm{~mJ} / \mathrm{cm}^{2}$ and $2.33 \mathrm{eV}$, respectively. Thick and thin curves are measured at $100 \mathrm{~ns}$ and $10 \mathrm{~ms}$. We call the $\sim 1.9 \mathrm{eV}$ band and the $\sim 2.5 \mathrm{eV}$ band as the I band and the PI band, respectively.

created by the photoexcitation, play an essential role in the PIPT process. In addition, we have found that the excitation spectra are qualitatively different between the I state and the PI phase, and interpreted the phenomena in terms of the siteselective excitation.

The Co-Fe cyanide film used in this investigation was prepared on $\mathrm{SnO}_{2}$-coated glass with an electrolysis process. The details of the synthesis procedure were described elsewhere. ${ }^{17,18}$ The chemical composition was $\mathrm{Na}_{0.35} \mathrm{Co}\left[\mathrm{Fe}(\mathrm{CN})_{6}\right]_{0.79} \cdot 3.7 \mathrm{H}_{2} \mathrm{O}$, and its thickness was $\approx 1 \mu \mathrm{m}$. The film shows the LS-to-HS transition at $T_{c \uparrow}$ $=306 \mathrm{~K}$ in the warming run, and at $T_{c \downarrow}=268 \mathrm{~K}$ in the cooling run. All the experiments were done above $\sim 200 \mathrm{~K}$, because the film shows the permanent PIPT below the temperature.

We first investigate the transient absorption spectra. The time-resolved spectroscopy was performed with a highly stable halogen lamp and a gatable CCD camera attached at the output stage of a grating monochrometer. The excitation light source was a second harmonics of the pulsed YAG laser $(2.33 \mathrm{eV}, 30 \mathrm{~Hz})$, whose pulse width was 3-5 ns. This photon energy corresponds to the charge-transfer excitation from the $\mathrm{Fe}^{2+}$ site to the $\mathrm{Co}^{3+}$ site. Figure 2 shows a prototypical example of the differential absorption spectra of $\mathrm{Na}_{0.35} \mathrm{Co}\left[\mathrm{Fe}(\mathrm{CN})_{6}\right]_{0.79} \cdot 3.7 \mathrm{H}_{2} \mathrm{O}$ at $260 \mathrm{~K}$. Soon after the photoexcitation, we observed two characteristic transient bands at $\sim 1.9 \mathrm{eV}$ (denoted as the I band) and at $\sim 2.5 \mathrm{eV}$ (PI band). The overall spectral profiles are consistent with Ref. 16. The latter band can be ascribed to the transient PI phase, ${ }^{16}$ because its spectral profile resembles that of the permanent PI phase measured at $90 \mathrm{~K}$ (not shown). Assuming $n_{\mathrm{PI}}=1$ after the permanent PIPT at $90 \mathrm{~K},{ }^{19}$ the site density $n_{\mathrm{PI}}$ of the PI phase at $260 \mathrm{~K}$ and at $t=100 \mathrm{~ns}$ is estimated to be 0.17 . Accordingly, the quantum efficiency of the PI phase is estimated to be $\approx 5$ sites/photon. On the other hand, the former band is ascribed to the $\mathrm{LS} \mathrm{Co}^{2+}$ species (I state in Fig. 1), ${ }^{16}$ judging from the much shorter lifetime $\tau_{\mathrm{I}}$ (vide infra). Both the absorption bands completely disappear at $10 \mathrm{~ms}$.

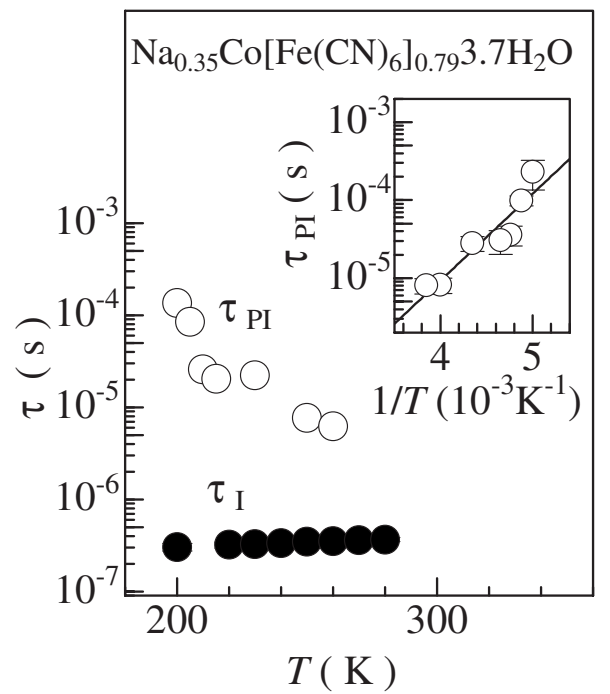

FIG. 3. Temperature dependence of the lifetimes for the PI band $\left(\tau_{\mathrm{PI}}\right.$ : open circles) and for the I state $\left(\tau_{\mathrm{I}}\right.$ : closed circles $)$. The pulse energy and the photon energy of the excitation light were $7 \mathrm{~mJ} / \mathrm{cm}^{2}$ and $2.33 \mathrm{eV}$, respectively. The inset shows the Arrhenius plot of $\tau_{\mathrm{PI}}$.

Now, let us proceed to the temporal evolution of the respective bands. The temporal behavior of the I band (PI band) was monitored with a continuous wave He-Ne laser at $1.96 \mathrm{eV}$ (YAG laser at $2.33 \mathrm{eV}$ ) and a digital oscilloscope (Textronics TDS5104B). In Fig. 3, we summarized the temperature dependence of the lifetimes, $\tau_{I}$ and $\tau_{\mathrm{PI}}$, for the respective bands. These lifetimes were almost constant irrespective of the pulse energy $I_{\text {pump }}\left(1 \mathrm{~mJ} / \mathrm{cm}^{2} \leq I_{\text {pump }}\right.$ $\leq 7 \mathrm{~mJ} / \mathrm{cm}^{2}$ ). The magnitude of $\tau_{\mathrm{PI}}$ increases as the temperature decreases. As shown in the inset, $\tau_{\mathrm{PI}}$ nearly obeys the Arrhenius relation with the activation energy $E_{\mathrm{ac}}=0.22 \mathrm{eV}$. Kawamoto et al. ${ }^{20}$ performed an ab initio cluster calculation on the $\mathrm{Co}(\mathrm{NC})_{5} \cdot \mathrm{H}_{2} \mathrm{O}$ cluster, and derived the total energy as a function of the $\mathrm{Co}-\mathrm{N}$ bond distance. Based on their calculation, the potential barrier between the $\mathrm{LS} \mathrm{Co}^{3+}$ state and the $\mathrm{HS} \mathrm{Co}^{2+}$ state is $\sim 0.5 \mathrm{eV}$. The calculated value is in good agreement with the experiment, taking account of the tendency that the cluster calculation usually overestimates the energy difference. On the other hand, the magnitude of $\tau_{\mathrm{I}}$ is much shorter than $\tau_{\mathrm{PI}}$, and is nearly constant $(\approx 300 \mathrm{~ns})$ irrespective of temperature.

Figure 4 shows the temporal evolution of $n_{\mathrm{PI}}$ at 215, 230, and $250 \mathrm{~K}$, monitored by the transmitting change at $2.33 \mathrm{eV}$. The fast component ( $\leq 20 \mathrm{~ns}$ ) seen in Fig. 4 is due to the stray of the excitation light. $n_{\mathrm{PI}}$ shows a characteristic behavior; at $215 \mathrm{~K}$, for example, $n_{\mathrm{PI}}$ gradually increases to $\approx 0.09$ within $\sim 1 \mu \mathrm{s}$, and then gradually decreases beyond $10 \mu \mathrm{s}$. Such a flow-in (flow-out) behavior of the $n_{\mathrm{PI}}-t$ curve strongly suggests that the PI phase is not directly created by the photoexcitation, but is gradually converted from the photocreated I state (see Fig. 1). In order to confirm this scenario, we analyzed the $n_{\mathrm{PI}}-t$ curves by a rate equation including the three energy levels, i.e., the LS phase, the I state, and the PI phase. The temporal evolution of $n_{\mathrm{PI}}(t)$ is expressed as 


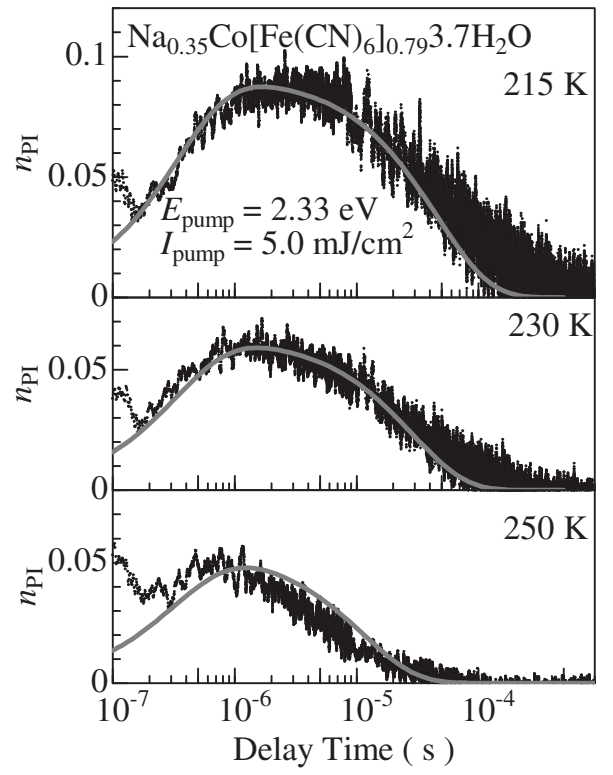

FIG. 4. Temporal evolution of the site density $n_{\mathrm{PI}}$ of the PI phase of the $\mathrm{Na}_{0.35} \mathrm{Co}\left[\mathrm{Fe}(\mathrm{CN})_{6}\right]_{0.79} \cdot 3.7 \mathrm{H}_{2} \mathrm{O}$ film at 215,230 , and $250 \mathrm{~K}$. The pulse energy and the photon energy of the excitation light were $5 \mathrm{~mJ} / \mathrm{cm}^{2}$ and $2.33 \mathrm{eV}$, respectively. The gray curves are the fitted results of the three-level model (see text).

$$
n_{\mathrm{PI}}(t)=\frac{\tau_{\mathrm{PI}}}{\tau_{\mathrm{PI}}-\tau_{\mathrm{I}}} n_{\mathrm{I}}^{0}\left(e^{-t / \tau_{\mathrm{PI}}}-e^{-t / \tau_{\mathrm{I}}}\right),
$$

where $n_{\mathrm{I}}^{0}$ is the initial site density of the I state after the photoexcitation. Here, we emphasize that only the magnitude $\left(\frac{\tau_{\mathrm{PI}}}{\tau_{\mathrm{PI}}-\tau_{\mathrm{I}}} n_{\mathrm{I}}^{0}\right)$ is the fitting parameter of Eq. (1), because we already know the temperature dependence of the lifetimes, i.e., $\tau_{\mathrm{I}}$ and $\tau_{\mathrm{PI}}$ (see Fig. 3). This simple model well reproduces the experimentally obtained $n_{\mathrm{PI}}-t$ curves, as shown by the gray curves in Fig. 4.

Based on these analyses, we propose a possible PIPT process of the Co-Fe cyanide. First, the charge-transfer excitation creates the $\mathrm{LS} \mathrm{Co}^{2+}-\mathrm{LS} \mathrm{Fe}^{3+}$ pair within $\sim 150 \mathrm{fs}^{21}$ forming the so-called Frank-Condon state. This FrankCondon state elongates the $\mathrm{Co}-\mathrm{Fe}$ bond distance, because the ionic radius $r_{\mathrm{LSCo}^{2+}}(=0.65 \AA)^{22}$ of the $\mathrm{LS} \mathrm{Co}{ }^{2+}$ ion is larger than $r_{\mathrm{LSCo}^{3+}}(=0.545 \AA)$ of the LS $\mathrm{Co}^{3+}$ ion. Actually, the lattice constant $a$ increases from $\approx 9.9 \AA$ in the LS phase $\left(\mathrm{Co}^{3+}-\mathrm{Fe}^{2+}\right.$ configuration $)$ to $\approx 10.3 \AA$ in the thermally induced charge-transferred phase $\left(\mathrm{Co}^{2+}-\mathrm{Fe}^{3+}\right.$ configuration $) .{ }^{10}$ The lattice relaxation is considered to spread around the charge-transferred pair to reduce the strain energy. In our preliminary ultrafast experiment, ${ }^{21}$ we found that the conversion efficiency is fairly reduced if we shorten the excitation light pulse from 2 to $3 \mathrm{~ns}$ to $100 \mathrm{fs}$ at the same photon energy $(\approx 0.3 \mathrm{eV})$ and photon number $(\approx 1.3$ $\times 10^{16} / \mathrm{cm}^{2} /$ pulse $)$. This observation implies that some state is formed within a few ns, which enhances the conversion efficiency. We think that the state is a cluster state of the charge-transferred pairs. As time exceeds $\sim 300 \mathrm{~ns}$, the LS $\mathrm{Co}^{2+}$ species change into the HS ones via the intersystem crossing. As a result, the metastable PI phase with the HS

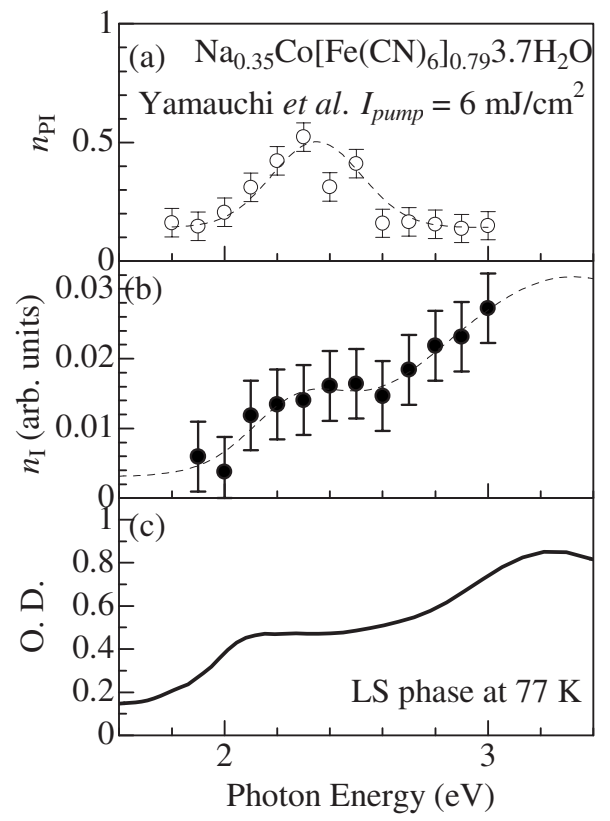

FIG. 5. (a) Excitation photon energy dependence of the site density $n_{\mathrm{PI}}$ of the PI phase of $\mathrm{Na}_{0.35} \mathrm{Co}\left[\mathrm{Fe}(\mathrm{CN})_{6}\right]_{0.79} \cdot 3.7 \mathrm{H}_{2} \mathrm{O}$ at $260 \mathrm{~K}$ (cited from Ref. 16). (b) Excitation photon energy dependence of the site density $n_{\mathrm{I}}$ of the I phase, monitored by the transmission change at $1.96 \mathrm{eV}$. (c) Absorption spectra at $77 \mathrm{~K}$ in the LS phase. Broken curves in (a) and (b) are merely the guide to the eyes.

$\mathrm{Co}^{2+}-\mathrm{LS} \mathrm{Fe}{ }^{3+}$ configuration is formed. We believe that the interaction between the charge-transferred pairs mediated by the lattice relaxation is essential for the formation of the transient PI phase, which means the charge-transferred cluster with cooperative lattice distortion. First, the quantum efficiency of the PI phase ( $\sim 5$ sites/photon) is much larger than unity, suggesting an energy transfer mechanism of the excess energy to the unexcited pairs. Second, the activation energy $E_{\mathrm{ac}}(=0.22 \mathrm{eV})$ of $\tau_{\mathrm{PI}}$ is consistent with the cluster calculation, ${ }^{20}$ suggesting that the lattice relaxation is important for the formation of the PI phase.

Finally, let us discuss the excitation spectra for the PI band and the I state (see Fig. 5). To obtain the excitation spectrum, the energy of the excitation light source $(3.49 \mathrm{eV}$, $30 \mathrm{~Hz}$ ) was converted with a nanosecond optical parametric oscillator (OPO) system. The excitation spectrum for the PI band [Fig. 5(a)] shows a single peak at $\sim 2.4 \mathrm{eV}$, which seems to be enhanced around the first peak $(\sim 2.1 \mathrm{eV})$ of the absorption $\alpha(\omega)$ spectrum of the LS phase [Fig. 5(c)]. This spectral difference between the excitation spectrum and the $\alpha(\omega)$ spectrum clearly excludes the conventional heating effect as an origin for the observed transient PIPT phenomenon. On the other hand, the excitation spectrum for the I state [Fig. 5(b)] rather traces the $\alpha(\omega)$ spectrum. In other words, the quantum efficiency increases at both the first $(\sim 2.1 \mathrm{eV})$ and the second $(\sim 3.2 \mathrm{eV})$ peak of the $\alpha(\omega)$ spectrum. These excitation spectra indicate that the formation of the I state is not the sufficient condition for the PIPT into the PI phase.

To deepen the argument, we first have to assign the first and second absorption peaks. The first peak, or the absorp- 
tion edge, was assigned to the charge-transfer (CT) excitation from the $\mathrm{Fe}^{2+}$ site to the $\mathrm{Co}^{3+}$ site, ${ }^{11}$ while the origin for the second peak remains unclear. Kawamoto et al. ${ }^{20}$ have performed an $a b$ initio calculation on the $\mathrm{Co}(\mathrm{CN})_{5} \cdot \mathrm{H}_{2} \mathrm{O}$ cluster and the $\mathrm{Co}(\mathrm{CN})_{6}$ cluster, and found that the resonant energy of the $\mathrm{CT}$ excitation significantly increases from $\sim 2.3 \mathrm{eV}$ for former cluster to $\sim 3.9 \mathrm{eV}$ for the latter cluster. They also found that the energy difference between the ground state (the LS phase) and the PI phase also increases from $\sim 0 \mathrm{eV}$ for the former cluster to $\sim 1.5 \mathrm{eV}$ for the latter cluster. Therefore, we assigned the first (second) absorption peak to the $\mathrm{CT}$ excitation on the $\mathrm{Co}(\mathrm{CN})_{5} \cdot \mathrm{H}_{2} \mathrm{O}$ cluster [the $\mathrm{Co}(\mathrm{CN})_{6}$ cluster]..$^{23}$ In an actual compound, however, there exists the spatial inhomogeniety of the vacancies, which causes the vacancy-rich and vacancy-poor regions.

Based on the above-motioned assignment, the observed excitation spectra (Fig. 5) are interpreted as follows. The photoexcitation of the first peak selectively induces the charge-transfer in the vacancy-rich regions. In this region, $\tau_{\mathrm{PI}}$ is considered to be much longer than $\tau_{\mathrm{I}}(\approx 300 \mathrm{~ns})$, because the energy difference between the LS phase and the PI phase is negligible. Then, the PI state should accumulate in the flow-in (flow-out) process (see Fig. 1). On the other hand, the excitation of the second peak excitation induces the charge-transfer in the vacancy-poor regions. In this region,
$\tau_{\mathrm{PI}}$ is comparable with $\tau_{\mathrm{I}}(\approx 300 \mathrm{~ns})$, since the energy difference is much larger. In this case, the fast relaxation of the PI state prevents the accumulation of the state. An analogous situation can be realized if we reduce the magnitude of $\tau_{\mathrm{PI}}$ (see Fig. 4). One may notice that the magnitude of $n_{\mathrm{PI}}$ is suppressed as temperature increases; $n_{\mathrm{PI}}$ decreases from $\approx 0.09$ at $215 \mathrm{~K}\left(\tau_{\mathrm{PI}}=20 \mu \mathrm{s}\right)$ to $\approx 0.05$ at $250 \mathrm{~K}\left(\tau_{\mathrm{PI}}=8 \mu \mathrm{s}\right)$.

In summary, we have investigated the dynamical behavior of PIPT of the Co-Fe cyanide, $\mathrm{Na}_{0.35} \mathrm{Co}\left[\mathrm{Fe}(\mathrm{CN})_{6}\right]_{0.79} \cdot 3.7 \mathrm{H}_{2} \mathrm{O}$, by means of the transient absorption spectroscopy. We observed a clear flow-in (flowout) behavior of $n_{\mathrm{PI}}$, which is quantitatively reproduced by the rate equation including three energy levels, i.e., the LS phase, the I state and the PI phase. Our observations suggest that the I state, whose lifetime is $\sim 300 \mathrm{~ns}$, plays an essential role in the PIPT process of the Co-Fe cyanide. We believe this is a first step to clarify how the single photoexcitation expands into a macroscopic phase.

This work was supported by a Grant-in-Aid for Scientific Research from the Ministry of Education, Couture, Sports and Science and from Iketani foundation. We also acknowledge the CREST project " $\mathrm{X}$-ray pinpoint structural measurement project" from Japan Science Technology Agency.
${ }^{1}$ Relaxzation of Exited States and Photoinduced Structural Transitions, edited by K. Nasu (Springer-Verlag, Berlin, 1977).

${ }^{2}$ J. Kusz, H. Spiering, and P. Gütlich, J. Appl. Crystallogr. 33, 201 (2000).

${ }^{3}$ J. Kusz, H. Spiering, and P. Gütlich, J. Appl. Crystallogr. 34, 229 (2001).

${ }^{4}$ M. Marchivie, P. Guionneau, J. A. K. Howard, G. Chastanet, J. F. Létard, A. E. Goeta, and D. Chasseau, J. Am. Chem. Soc. 124, 194 (2002).

${ }^{5}$ N. Huby, L. Guérin, E. Collet, L. Toupet, J.-C. Ameline, H. Cailleau, T. Roisnel, T. Tayagaki, and K. Tanaka, Phys. Rev. B 69, 020101(R) (2004).

${ }^{6}$ O. Sato, T. Iyoda, A. Fujishima, and K. Hashimoto, Science 272, 704 (1996).

${ }^{7}$ O. Sato, Y. Einaga, A. Fujishima, and K. Hashimoto, Inorg. Chem. 38, 4405 (1999).

${ }^{8}$ H. Tokoro, T. Matsuda, K. Hashimoto, and S. Ohkoshi, J. Appl. Phys. 97, 10M083 (2005).

${ }^{9}$ Y. Moritomo, M. Hanawa, Y. Ohishi, K. Kato, M. Takata, A. Kuriki, E. Nishibori, M. Sakata, S. Ohkoshi, H. Tokoro, and K. Hashimoto, Phys. Rev. B 68, 144106 (2003).

${ }^{10}$ M. Hanawa, Y. Moritomo, A. Kuriki, J. Tateishi, K. Kato, M. Takata, and M. Sakata, J. Phys. Soc. Jpn. 72, 987 (2003).

${ }^{11}$ N. Shimamoto, S. Ohkoshi, O. Sato, and K. Hashimoto, Inorg. Chem. 41, 678 (2002).

${ }^{12}$ X. J. Liu, Y. Moritomo, M. Ichida, A. Nakamura, and N. Kojima,
Phys. Rev. B 61, 20 (2000).

${ }^{13}$ H. Matsuzaki, T. Matsuoka, H. Kishida, K. Takizawa, H. Miyasaka, K. Sugiura, M. Yamashita, and H. Okamoto, Phys. Rev. Lett. 90, 046401 (2003).

${ }^{14}$ H. Matsuzaki, W. Fujita, K. Awaga, and H. Okamoto, Phys. Rev. Lett. 91, 017403 (2003).

${ }^{15}$ S. Koshihara, Y. Tokura, K. Takeda, and T. Koda, Phys. Rev. Lett. 68, 1148 (1992).

${ }^{16}$ T. Yamauchi, A. Nakamura, Y. Moritomo, T. Hozumi, K. Hashimoto, and S. Ohkoshi, Phys. Rev. B 72, 214425 (2005).

${ }^{17}$ Y. Sato, S. Ohkoshi, and K. Hashimoto, J. Appl. Phys. 92, 4834 (2002).

${ }^{18}$ O. Sato, Y. Einaga, T. Iyoda, A. Fujishima, and K. Hashimoto, J. Electrochem. Soc. 144, L11 (1991).

${ }^{19} \mathrm{We}$ investigated the differential absorption spectrum as a function of photoirradiation time at $90 \mathrm{~K}$. The spectral change was almost saturated in $30 \mathrm{~s}$.

${ }^{20}$ T. Kawamoto, Y. Asai, and S. Abe, Phys. Rev. Lett. 86, 348 (2001).

${ }^{21}$ Kamioka et al. (unpublished).

${ }^{22}$ R. D. Shannon, Acta Crystallogr., Sect. A: Cryst. Phys., Diffr., Theor. Gen. Crystallogr. A32, 1751 (1976).

${ }^{23}$ The probability to find the $\mathrm{Co}(\mathrm{CN})_{5} \cdot \mathrm{H}_{2} \mathrm{O}$ cluster [the $\mathrm{Co}(\mathrm{CN})_{6}$ cluster] is $0.32(0.14)$, if we assume the random distribution of the $\left[\mathrm{Fe}(\mathrm{CN})_{6}\right]$ vacancies. 